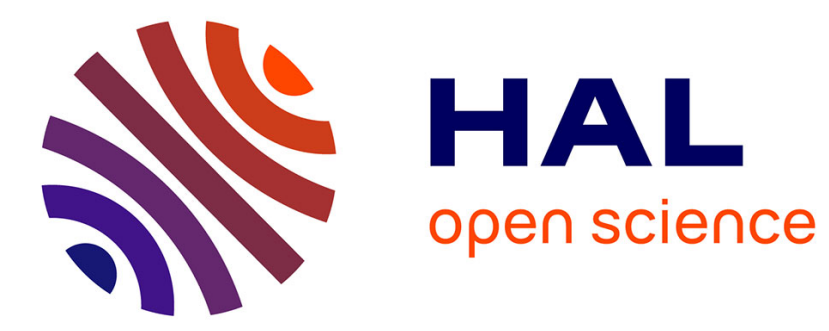

\title{
Montpellier Infectious Diseases -Pôle Rabelais (MID) 3rd annual meeting (2014)
}

Sébastien Besteiro, Anne Béatrice Blanc-Potard, Matteo Bonazzi, Laurence

Briant, Nathalie Chazal, Emmanuel Cornillot, Gaëlle Lentini, Roy Matkovic, Armen Sanosyan, Edouard Tuaillon, et al.

\section{To cite this version:}

Sébastien Besteiro, Anne Béatrice Blanc-Potard, Matteo Bonazzi, Laurence Briant, Nathalie Chazal, et al.. Montpellier Infectious Diseases -Pôle Rabelais (MID) 3rd annual meeting (2014). Infection, Genetics and Evolution, 2015, 32, pp.161-164. 10.1016/j.meegid.2015.03.007 . hal-02149411

\section{HAL Id: hal-02149411 \\ https://hal.science/hal-02149411}

Submitted on 6 Jun 2019

HAL is a multi-disciplinary open access archive for the deposit and dissemination of scientific research documents, whether they are published or not. The documents may come from teaching and research institutions in France or abroad, or from public or private research centers.
L'archive ouverte pluridisciplinaire HAL, est destinée au dépôt et à la diffusion de documents scientifiques de niveau recherche, publiés ou non, émanant des établissements d'enseignement et de recherche français ou étrangers, des laboratoires publics ou privés. 


\section{Conference report}

\section{Montpellier Infectious Diseases - Pôle Rabelais (MID) 3rd annual meeting (2014)}

Sébastien Besteiro ${ }^{a}$, Anne Blanc-Potard ${ }^{a}$, Matteo Bonazzi ${ }^{b}$, Laurence Briant ${ }^{b}$, Nathalie Chazal $^{b^{*}}$, Emmanuel Cornillot ${ }^{b}$, Gaëlle Lentini ${ }^{a}$, Roy Matkovic ${ }^{b}$, Armen Sanosyan $^{c}$, Edouard Tuaillon ${ }^{c}$, Philippe Van de Perre ${ }^{c}$.

${ }^{a}$ Dynamique des Interactions Membranaires Normales et Pathologiques (DIMNP) UMR 5235 CNRS Université de Montpellier, Montpellier, France.

${ }^{b}$ Centre d'études d'agents Pathogènes et Biotechnologies pour la Santé (CPBS), FRE 3689 CNRS Université de Montpellier, Montpellier, France.

${ }^{c}$ Inserm U1058, Université Montpellier \& CHRU de Montpellier, Département de BactériologieVirologie \& Institut de Recherche en Biothérapie, Montpellier, France

*Corresponding author: nathalie.chazal@cpbs.cnrs.fr

Address correspondence to: Nathalie Chazal, CPBS, FRE 3689 CNRS-UM, 1919 route de Mende, 34293 Montpellier cedex 5, France

\section{Keywords:}

Infectious diseases

Translational research

Multidisciplinary approaches 


\section{Abstract}

For the third time, teams belonging to the "Montpellier Infectious Diseases" network in the Rabelais BioHealth Cluster held their annual meeting on the $27^{\text {th }}$ and $28^{\text {th }}$ of November in Montpellier, France. While the 2012 meeting was focused on the cooperation between the local force tasks in biomedical and medical chemistry and presented the interdisciplinary research programs designed to fight against virus, bacteria and parasites, the 2014 edition of the meeting was focused on the translational research in infectious diseases and highlighted the bench-to-clinic strategies designed by academic and private research groups in the Montpellier area.

\section{Introduction}

Infectious diseases, which cause 15 million deaths per year, represent a major challenge to human society. Recent advances on human threatening infectious diseases have emphasized that the setup of effective management and the development of efficient strategies to eradicate, or control, infectious diseases propagation, both rely on basic research and on the coordination of translational approaches with medical services and industrial partners.

The Rabelais BioHealth Cluster is a public-funded structure aimed at coordinating and promoting new ideas, science events, communication, research excellence and partnerships, at the biology/health interface for research, healthcare and training institutions located in the Montpellier area. It was created by the local scientific, academic and professional public institutions of Montpellier, together with the local Regional University Hospital Centers of Montpellier and Nîmes and the Regional Cancer Institute of Montpellier (ICM). Besides its local federating functions, the Rabelais BioHealth Cluster has also a national and 
international role in the field of Biology and Health. Moreover, it fosters the collaborative network with its industrial partners; composed of locally implanted, innovative and dynamic companies recognized as European leaders in health industry. Currently, the "Infectiology" axis of the Rabelais BioHealth Cluster brings together 14 academic laboratories comprising more than 200 researchers. They are microbiologists, investigating different areas of bacteriology, virology, parasitology; but also immunologists, cell biologists and epidemiologists interested in infectious diseases. Physicians involved in clinical research are also part of this research network.

The ambition of the Montpellier Infectious diseases - Rabelais BioHealth Cluster 2014 (http://mid2014.sciencesconf.org) - was to bring together teams working on infectious diseases and promote interconnections and collaborations between research projects. In addition, the objectives were to pinpoint areas of scientific excellence and originality and develop them in a coordinated way, thereby responding to major international challenges regarding infectious diseases.

The conference took place at the Genepolys convention center, on November $27^{\text {th }}$ and $28^{\text {th }}, 2014$ and was attended by 120 scientists of the Rabelais BioHealth Cluster research network. This event highlighted the opportunity to affirm the visibility of a network that aims to become an international reference in the field of infectious diseases. The meeting schedule was based on 3 plenary sessions (1 - Infection, Transmission and Drug Design; 2 Infection, Immunity and Regulation; 3 - Epidemiology, Clinical Trials and Emerging Pathogens), with 19 plenary speakers and 21 posters. This meeting showed several examples of excellent basic science, but also proved the capacity of local researchers to develop interdisciplinary programs with the industry, and to promote integrated anti-infectious approaches targeting a broad range of host-pathogen interactions. 


\section{Session summaries}

\subsection{Infection, Transmission and Drug Design}

In this session, three virology-related talks provided a good overview of how a better understanding of the interactions between host and virus determinants can lead to the design of potential therapeutic strategies. First, Pierre Corbeau (IGH, UPR1142) provided a excellent description of a differential Human Immunodeficiency Virus (HIV) receptor isoform expression and function that can lead to a specific inhibition of viral entry, with a limited impact on the receptor's primary physiological role for the host. CXCR4 is a chemokine receptor with multiple functions in embryonic development, immune system and cell homeostasis maintenance. In addition to the main viral receptor CD4, there are several potential co-receptors such as CCR5, used by R5 HIV strains, and CXCR4 used by X4 HIV strains that may emerge in the later stages of the disease. Two isoforms of CXCR4, CXCR4-A and CXCR4-B, have been described in humans. Pierre Corbeau's team has shown that R5 strains favor CXCR4-B expression in infected host cells and thereby the subsequent emergence of X4 strains. Actually, the work presented at MID 2014 supports that CXCR4-B, but not CXCR4-A, mediates an efficient HIV-1 X4 entry and productive infection. However, as both isoforms have a similar chemokine binding activity, the authors have achieved a specific RNA interference-mediated down-regulation of CXCR4-B that impairs the propagation of HIV-1 X4, without interfering with the chemotactic activity and physiological function of CXCR4-A. This opens the possibility for a CXCR4-B-targeting gene-therapeutic approach to control particular stages of HIV-1 infection. The work presented by JeanFrançois Guichou (CBS, UMR1054) focused on the rational design of inhibitors of Hepatitis C Virus (HCV) replication by targeting a host enzyme. HCV is a positive-strand RNA virus for which host cyclophilins are considered to play an important role in viral replication. 
Cyclophilins are abundant proteins that facilitate protein folding through a prolyl isomerase activity: they allow the isomerization of peptide bonds, from a trans to a cis form, at proline residues. A fragment-based screening approach was used to design specific inhibitors of cyclophilin D. This strategy is based on the identification of small chemical fragments, which may bind only weakly to the biological target and then combining them to produce a lead with a higher affinity. X-ray crystallography and nuclear magnetic resonance were used in combination to identify potential hits and solve their crystallographic structures together with cyclophilin D. After optimization, proline-mimetic lead compounds were obtained with an IC50 of $10 \mathrm{nM}$ on cyclophilins D and A in vitro, and an EC50 of $100 \mathrm{nM}$ on HCV replication in cellulo. This work demonstrates the potential of fragment-based screening approaches for identifying new anti-viral compounds. Another study was presented by Patrick Bron (CBS, UMR1054) and focused on the virus side of host/pathogen interactions for better characterising determinants involved in the transmission of picoRNA-like plant viruses. The genus Nepovirus comprises the Arabis Mosaic Virus (ArMV) and the Grapevine FanLeaf Virus (GFLV) which are some of the most destructive pathogens of grapevine worldwide. Transmission of the ArMV is dependent on vectors such as soil-borne nematode Xiphinema diversicaudatum, while GFLV depends on a different nematode species, $\mathrm{X}$. index. The viruses consist of a bipartite RNA genome encapsidated into a $30 \mathrm{~nm}$ icosahedral viral particle formed by 60 copies of a single capsid protein (CP). Given that ArMV and GFLV CP share a significant identity at the amino-acid level, while the viruses are transmitted by two different nematode species, comparison of their capsid structures is potentially important to understand virus-vector transmission specificity. The work presented at the symposium combined X-ray crystallography, cryo-electron microscopy, single particle analysis and molecular dynamics methods, which led to the identification of a charged pocket in the CP 
involved in transmission. Two other presentations from the session focused on parasitic protists, dealing with fundamental aspects of the replication of the pathogens or essential biochemical pathways including potential drug targets. The mitotic process is diverse among protists. Understanding how intracellular pathogens divide and replicate can obviously help discovering new avenues for potential therapeutic intervention. Patrick Bastien (MIVEGEC, IRD UR224) described new insights into chromosomal DNA replication and segregation in Leishmania parasites. Unlike most eukaryotic cells for which the even transmission of the genetic material to the two daughter cells is strictly controlled, Leishmania has a unique ploidy organisation termed 'mosaic aneuploidy'. This particular chromosomal configuration could confer some advantages to the parasite to adapt to various growth conditions encountered during its life cycle. The work presented combines the use of imaging and molecular techniques to describe the replication process in Leishmania, but also takes advantage of related kinetoplastid Trypanosoma brucei, which is more amenable to genetic manipulation, to identify factors involved in controlling ploidy in these parasites. These include TbMlp2, a member of the nucleopore complex which might be involved in the dynamics of chromosomal segregation in kinetoplastids. The work presented by Alicia Contet (DIMNP, UMR5235) is related to Plasmodium falciparum, another parasitic protist, and the causative agent of malaria. During its division process inside human erythrocytes, the parasite builds up a significant amount of membranes to replicate its daughter cells. It is thus particularly dependent on de novo phospholipids synthesis. More precisely, the biosynthesis of phosphatidylcholine (PC) and phosphatidylethanolamine (PE) involve CDPcholine and CDP-ethanolamine-dependent pathways. As a striking example, phosphocholinecytidylyltransferase (PfCCT) is a crucial and limiting step enzyme for PC biosynthesis in the parasite. The 3D crystal structure of the C-terminal catalytic domain of 
PfCCT in complex with its reaction product CDP-choline was obtained at a resolution of 2.4

Å. A combination of virtual screenings of commercial compounds libraries using docking tools, as well as a fragment-based drug design strategy have both been used to identify molecules specifically targeting PfCCT. These will serve as a starting point for subsequent rational drug design to generate inhibitors of this key parasite enzyme.

\subsection{Infection, Immunity and Regulation}

The main conference of this session was given by an invited speaker, Priscille Brodin (Pasteur Institute, Lille). P. Brodin's team has recently achieved an impressive work on critical features of Mycobacterium tuberculosis within live target cells, both on the pathogen and host sides, by developing novel approaches for the high-content imaging of $M$. tuberculosis-infected cells. This has proven extremely successful in the fight against antibiotic resistance. Initial studies were performed on infected macrophages, which are reservoirs for replicating Mycobacteria during tuberculosis infections. High-content imaging technologies offer a high-throughput method to quantify a drug's ability to inhibit $M$. tuberculosis intracellular invasion and multiplication in host cells and several promising compounds have been identified. Next, extrapulmonary dissemination of $M$. tuberculosis and the role of non-phagocytic cells, two poorly understood aspects of infection have been discussed and P. Brodin has reported the identification of host genes involved in the internalisation of $M$. tuberculosis within epithelial cells. Finally, P. Brodin's team has contributed to an unexpected major discovery on $M$. ulcerans toxin showing that the secreted mycobacterial mycolactone induces analgesia in buruli ulcer by targeting the angiotensin pathways. It was previously thought that analgesia was ensured by direct nerve cell destruction but M. ulcerans-induced hypoesthesia is actually achieved through a specific 
neurological pathway that involves type 2 angiotensin II receptors, leading to potassiumdependent hyperpolarization of neurons. This leading conference was then followed by two short talks on emerging bacterial pathogens, M. abscessus and Burkholderia cepacia complex species that are found in cystic fibrosis patients. In both studies, zebrafish embryos, which have an innate immune system very similar to that of humans, are used as a novel and powerful infection model to decipher the molecular and cellular mechanisms involved in bacterial pathogenicity. Mycobacterium abscessus (Mabs) is an emerging rapid-growing mycobacteria causing severe lung infections, particularly in cystic fibrosis patients. Rough variants, characterized by the loss of surface glycopeptidolipids, are involved in severe clinical forms although the underlying physiopathological mechanisms remain obscure. The talk presented by Iman Halloum (DIMNP, UMR CNRS 5235, Montpellier) focused on the identification and characterization of a new Mabs determinant required for intracellular survival and pathogenicity. A rough mutant of MABS_4780 was constructed. This strain exhibited a higher susceptibility to thiacetazone, a second-line antitubercular drug, compared to the parental strain and higher sensitivity to detergents, presumably due to uncharacterised alterations of cell wall composition/structure. The mutant failed to replicate intracellularly in amoebas (a potential environmental reservoir of Mabs) and macrophages. In addition, in the zebrafish embryo model, the MABS_4780 mutant strain is extremely attenuated. These findings demonstrate the unanticipated role of MABS_4780 in physiopathology of Mabs infection, emphasizing its potential as an attractive drug target. Chronic respiratory infection in cystic fibrosis patients caused by bacteria belonging to the Burkholderia cepacia complex (Bcc) can be symptom-free, but often cause pulmonary exacerbation with progressive worsening of lung function, sometimes resulting in acute fatal necrotizing pneumonia and sepsis. The reasons for these unpredictable, sudden transitions 
are not understood. In zebrafish embryos, some strains, as B. cenocepacia $\mathrm{K} 56-2$, are highly virulent and cause a rapidly fatal systemic inflammatory infection. In contrast, embryos can control infection with other strains, such as B. stabilis LMG14294, which cause a persistent infection. The talk given by Jennifer Mesureur (INSERM U1047, Nîmes) focused on the host immune response and macrophage behavior observed during $B$. cepacia complex infection in zebrafish embryos. To better understand the host molecular basis for B. cenocepacia K562 or B. stabilis LMG14294 infection outcomes, a global host transcriptome analysis during different stages of both infection types has been performed. J. Mesureur discussed how the observed changes in gene expression level correlated with the acute and persistent infection phenotypes, and knock-down approaches showed the role of several pro-inflammatory cytokines and TLR signaling proteins in infection. The following presentation by Veronique Jubier-Maurin (CPBS, FRE3689) focused on the role of the transcriptional regulator RegA in the establishment of Brucella suis persistent infections. Brucella spp. is the bacterial genus responsible for brucellosis, a zoonotic disease that affects livestock animals with severe consequences on agriculture. It has been reported that during intramacrophagic replication and chronic infection, Brucella organisms are exposed to oxygen-depleted environments. In response to these environmental challenges Brucella has developed a highly flexible metabolism. Previous work from Dr. Jubier-Maurin has highlighted an essential role of the $B$. suis two-component system RegB/RegA in the coordinated control of oxidative respiration and denitrification, which are essential for virulence and/or persistence in vivo. To better investigate the role of RegA in response to low oxgen, the team has developed an interesting in vitro model, which allows the controlled depletion of oxygen from B. suis cultures. This has allowed global transcriptome and proteome analyses of $B$. suis at the time point where anaerobiosis become established and demonstrated that RegA is potentially involved in the 
regulation of $12 \%$ of $B$. suis genes. These include genes involved in bacterial replication as well as energy production, suggesting that globally, RegA may control Brucella transition from a replicative to a persistent state. Ayappasamy Sudalaiyadum Perumal (CPBS, FRE3689) gave the closing presentation for this session. His work focuses on the characterization of $\mathrm{RbpA}$, a master regulator of gene expression in $M$. tuberculosis. $\mathrm{RbpA}$ is a non-DNA binding activator protein that regulates transcription by helping RNA polymerase to unwind the promoter DNA. It is predominantly found in actinomycetes and it can increase the tolerance to antibiotics, including Rifampicin, the most common antibiotic used in the treatment of tuberculosis. Using in vitro transcription systems (IVTS) and electrophoretic mobility shift assays (EMSA), the team has observed that the requirement of RbpA in gene activation is specific for $\sigma$ factors, namely $\sigma A$ and $\sigma B$, and the RbpA-specific sequence in the promoter region of the genes regulated by RbpA have been mapped. Importantly, run-off microarrays (ROMA) is being currently used to obtain a global mapping of RbpA specific sequences. These studies can lead to the development of new antibacterial drugs that target RbpA.

\subsection{Epidemiology, Clinical Trials and Emerging Pathogens}

An important goal of research programs on infectious diseases is to identify a molecular target leading to the development of new drugs, new candidates that would undergo and pass the step of clinical trials to become a brand new efficient treatment. This medication will then be proposed to the affected population where the microorganism is proliferating. This session deals with the epidemiology of several viruses threatening southern countries and spreading to developing ones, thus becoming a worldwide concern. Xavier de 
Lamballerie ( $\mathrm{CHU}$, Marseille) opened this session as an invited speaker by presenting a promising strategy to develop a safe, live attenuated, RNA virus vaccine in a cost effective manner. His team demonstrated that random codon re-enconding which consists in modifying coding regions in the virus genome could confer a decrease in the replicative fitness. Indeed, despite emergence of synonymous and-non synonymous mutations in other genomic regions, no codon reversion could have been detected after many passages in cell cultures. Such result has been obtained with the Chikungunya Virus for which no vaccine exists yet, thus opening a new way to generate a preventing medication that would establish a long term protection against the Chikungunya fever. Encouraging methods for evaluating the nature and progression of these viral epidemics, and the strategies designed to counter them, are described here after. First, Patrice Ravel (CPBS-CNRS-Université de Montpellier) described the methods used to model the spread of Human Enterovirus A responsible of the Hand, foot and mouth disease (HFMD) between 2011 and 2012 in Northern Vietnam. His first approach consisted in determining and understanding the high level of diversity of the clinical signs, using the three most solid clinical quantitative parameters; i.e., age, severity score and delay from onset to admission from a clinical data set of the 10000 observed cases. A hierarchical classification was used to cluster the patients into groups of common symptoms and analyze their distribution over the span of the epidemic. The second approach consisted in analyzing the spread of HFMD according to time and space. The commune is the smallest subdivision of Hai Phong City. Using the commune for geographical localization and the time distribution of the detected cases, recording was done in Geographic Information System (QGIS). The data were used to create a graph that conceptualized the outbreak of HFMD. Numerical analyses were investigated to determine some group of communes that presented similarities concerning the spatial dynamic of 
HFMD. Then, Christina Psomas (CHU Montpellier) characterized the immune activation of a cohort of 120 HIV-infected adults and highly active antiretroviral therapy (HAART) treated patients and defined various subpopulations. She made clear that a better understanding of the links between causes, patterns, and consequences of immune activation in virologic responders might pave the way to the identification of predictive markers for specific comorbidities, and to an etiologic and/or symptomatic immunosuppressive therapeutic approach tailored to each subpopulation of patients. Another study by Charlotte Boullé (TransVIHMI - Université Cheikh Anta Diop (Dakar, Sénégal) - Universtié Yaoundé 1 (Cameroun) - Université de Montpellie - IRD) pinpointed the gender differences in adherence and response to antiretroviral treatment in the Stratall trial led in rural district hospitals in Cameroon. Her results showed that, in spite of a higher body mass index, hemoglobin level, intake of the efavirenz antiviral drug, African men are more vulnerable to anti-retroviral therapy failure than women and that the male vulnerability extends beyond adherence issues. Future studies are still required to determine the causes for this vulnerability in order to optimize HIV care. The two last talks concerned Chikungunya virus (CHIKV), an emerging Arbovirus that has also been recently found to circulate in the Northern countries causing epidemics. First of all, Laurence Briant (CPBS-CNRS-Université de Montpellier) reported that $\mathrm{CHIKV}$ rapidly elicits an innate immune response in keratinocyte cells after puncture of the skin by a blood-feeding infected Aedes mosquito leading to the enhanced transcription of type I/II and type III interferon genes. Concomitantly, her team smartly showed that despite viral particles internalization into Rab5-positive endosomes and efficient fusion of virus and cell membranes, keratinocytes poorly replicate CHIKV as attested by absence of nonstructural proteins and genomic RNA synthesis. Accordingly, and in contrast to other Arboviruses such as Dengue Virus (DV) or West Nile Virus (WNV), human 
keratinocytes behave as an antiviral defense against CHIKV infection rather than as a primary targets for initial replication. Peeraya Ekchariyawat (IRD-MIVEGEC) explained that CHIKV and WNV, despite belonging to distinct viral families, lead to a common antiviral signature in primary human dermal fibroblasts attesting for the upregulation of interferon signaling pathways and leading to an enhanced expression of IFN- $\beta$, interleukines and chemokines. Remarkably, both WNV and CHIKV enhance IL-1 $\beta$ genes expression and induce maturation of caspase-1, indicating the capacity of these pathogens to elicit activation of the inflammasome program in resident skin cells. Her analysis further demonstrated that activation of the inflammasome limits chikungunya virus replication in human dermal fibroblasts, indicating that dermal fibroblasts, in agreement with Laurence Briant's data, contribute to the pro-inflammatory and antiviral microenvironment created at the skin level in the early stages of interaction with arboviruses.

\section{Acknowledgements}

The organizing committee of the 3rd annual meeting thanks the University of Montpellier, the CHRUs of Montpellier-Nîmes, the national research institutions, the "Pôle Rabelais", Biocampus Montpellier, Epigenmed, Binder, Roth, Sartorius, Beckman Coulter, Life Technologies for supporting or sponsoring of this meeting. 